\section{Tight junctions in Hailey-Hailey and Darier's diseases}

\author{
Laura Raiko, ${ }^{1}$ Pekka Leinonen, ${ }^{2,3}$ \\ Päivi M. Hägg, ${ }^{3}$ Juha Peltonen, ${ }^{4}$
}

Aarne Oikarinen, ${ }^{3}$ Sirkku Peltonen'

'Department of Dermatology, University of Turku and Turku University Central

Hospital, Turku, Finland; ' 2 Department of

Anatomy and Cell Biology and

${ }^{3}$ Department of Dermatology, University

of Oulu, Oulu, Finland; ${ }^{4}$ Institute of

Biomedicine, Department of Anatomy,

University of Turku, Turku, Finland

\section{Abstract}

Hailey-Hailey disease (HHD) and Darier's disease (DD) are caused by mutations in $\mathrm{Ca}^{2+}$ ATPases with the end result of desmosomal disruption and suprabasal acantholysis. Tight junctions (TJ) are located in the granular cell layer in normal skin and contribute to the epidermal barrier. Aberrations in the epidermal differentiation, such as in psoriasis, have been shown to lead to changes in the expression of TJ components. Our aim was to elucidate the expression and dynamics of the TJ proteins during the disruption of desmosomes in HHD and DD lesions. Indirect immunofluorescence and avidin-biotin labeling for TJ, desmosomal and adherens junction proteins, and subsequent analyses with the confocal laser scanning microscope were carried out on 14 HHD and 14 DD skin samples. Transepidermal water loss (TEWL) was measured in normal and lesional epidermis of nine HHD and eight DD patients to evaluate the function of the epidermal barrier in HHD and DD skin. The localization of TJ proteins claudin-1, claudin-4, Z0-1, and occludin in perilesional HHD and DD epidermis was similar to that previously described in normal skin. In HHD lesions the tissue distribution of Z0-1 expanded to the acantholytic spinous cells. In agreement with previous findings, desmoplakin was localized intracellularly. In contrast claudin-1 and Z0-1 persisted in the cell-cell contact sites of acantholytic cells. TEWL was increased in the lesional skin. The current results suggest that TJ components follow different dynamics in acantholysis of HHD and DD compared to desmosomal and adherens junction proteins.

\section{Introduction}

Hailey-Hailey disease (HHD, OMIM 16960) and Darier's disease (DD, OMIM 124200) are rare blistering skin diseases inherited as an autosomal dominant trait. HHD results in mutations in the ATP2C1 gene, which encodes the Golgi secretory pathway $\mathrm{Ca}^{2+} / \mathrm{Mn}^{2+}$ ATPase (hSPCA1), ${ }^{1,2}$ DD is caused by mutations in the ATP2A2 gene encoding $\mathrm{Ca}^{2+}$-ATPase type 2 (SERCA2, sarcoplasmic/endoplasmic reticulum $\mathrm{Ca}^{2+}$-transport ATPase isoform 2b). ${ }^{3}$ The most prominent common epidermal histological feature of DD and HHD is suprabasal acantholysis, which results from desmosomal disintegration. In addition DD and HHD epidermis show differentiation and keratinization defects. ${ }^{4-6}$ Specifically transition of keratin 14 to keratin 10 is abnormal as demonstrated by the presence of suprabasal keratinocytes expressing both cytokeratins that usually are exclusive in normal epidermis. ${ }^{6}$ Although the primary abnormalities in calcium metabolism in HHD and DD are known, the sequence of events leading to acantholysis is not understood fully yet.

In normal human epidermis, tight junctions (TJ) are located in the granular layer ${ }^{7,8}$ where they contribute to the epidermal barrier function, especially the diffusion of water from inside out. ${ }^{9-11}$ The most important transmembrane proteins of epidermal $\mathrm{TJ}$ are the members of the claudin family; namely claudins- 1 and -4 , which are expressed in all vital epidermal layers. ${ }^{8,12}$ Transmembrane protein occludin and intracellular linking molecule zonula occludens protein 1 (ZO-1) are restricted to the granular layer normally. ${ }^{7}$ Previously we have shown that abnormal epidermal differentiation is associated with disturbances in distribution of TJ proteins. ${ }^{13}$ Examples of aberrant differentiation include psoriatic epidermis and hypertrophic edges of healing blisters, which show spreading of ZO1 and occludin to the acanthotic spinous cell layers. ${ }^{7,13,14}$ Up-regulation of Z0-1 and occludin is reversible and disappears during the healing of the psoriasis lesion. ${ }^{13}$ Regulation of TJ proteins in epidermis is not well known but the presence of $\mathrm{TJ}$ in the granular cell layers suggests that the prerequisite for the formation of TJ is high extracellular calcium concentration. In fact studies on cultured keratinocytes have shown that development of TJ is calcium-inducible. ${ }^{7,15}$ We have demonstrated recently that in HHD and DD lesions the calcium concentration in the basal layer is lower than in normal skin. ${ }^{6}$ In addition to being decreased in the basal cells in the lesional $\mathrm{HH}$ and DD epidermis, the calcium content was decreased in non-lesional DD epidermis, probably linking to the keratinization defect seen especially in DD. ${ }^{6}$

The localization of adherens junction and desmosomal proteins in acantholytic lesions of DD and HHD has been studied in detail previously. ${ }^{16}$ However the dynamics of TJ pro-
Correspondence: Laura Raiko, Department of Dermatology, University of Turku, 20520 Turku, Finland. E-mail: laura.raiko@utu.fi

Key words: adherens junction, claudin, DarierWhite disease, tight junction, zonula occludens protein 1 .

Acknowledgments: we thank Dr Lauri Talve, Department of Pathology, Turku University Central Hospital for providing the archival tissue material. This study was supported financially by grants from the Finnish Medical Foundation, Finnish Cultural Foundation, Academy of Finland, the Turku University Foundation, the Southwest Finland Hospital District, and Northern Ostrobothnia Hospital District, Finland.

Contributions: LR, immunolabeling, confocal microscopy, writing the manuscript, and designing the figures; PL, TEWL measurements, immunolabeling for frozen sections; PMH, obtaining patient samples in Oulu, TEWL measurements; JP, writing the manuscript and supervising the work; $\mathrm{AO}$, patient samples and supervising the work in Oulu; SP, coordinating the work and participating in all parts except TEWL measurements.

Conflict of interest: the authors reported no conflicts of interest.

Received for publication: 1 October 2009.

Revision received: 2 November 2009.

Accepted for publication: 3 November 2009.

This work is licensed under a Creative Commons Attribution 3.0 License (by-nc 3.0).

(C) Copyright L. Raiko et al., 2009

Licensee PAGEPress, Italy

Dermatology Reports 2009; 1:e1

doi:10.4081/dr.2009.e1

teins has not been studied in acantholysis. Studies on epidermal cadherin knockout mice suggest the importance of cadherins in assembly and/or stability of TJ and desmosomes. ${ }^{17,18}$ Thus desmosomes and TJ seem to be at least partly co-regulated.

The aims of this study were: (1) to elucidate the role of $\mathrm{TJ}$ in the epidermal barrier in blistering disease; (2) to study the localization of TJ in an abnormal epidermal calcium gradient; (3) to see whether the aberrant differentiation in DD has an effect on TJ in the analogy of psoriatic skin and healing wounds; (4) to compare the dynamics of TJ components to that of desmosomal and adherens junction proteins at the cellular level in the acantholytic process. Specifically we investigated the localization of $\mathrm{TJ}$ components claudin-1, claudin- $4, \mathrm{ZO}-1$, and occludin in frozen and paraffin-embedded skin of $14 \mathrm{HHD}$ and 14 DD patients using commercial antibodies for indirect immunofluorescence and 
avidin-biotin immunolabeling. Co-localization of TJ proteins with adherens junction and desmosomal components was demonstrated using the confocal laser scanning microscope. In addition transepidermal water loss (TEWL) was elucidated by measuring TEWL in apparently normal and lesional $\mathrm{HH}$ and DD skin. The results showed that in both diseases the tissue localization of TJ proteins remained apparently normal in the lesional and perilesional epidermis, while acantholysis was associated with changes in the distribution of TJ proteins at the cellular level.

\section{Materials and Methods}

\section{Biopsy samples}

The skin biopsies were taken at the Department of Dermatology, Turku University Central Hospital and the Department of Dermatology, Oulu University Hospital, Finland, with approval of the Ethical Committee of the Southwest Finland Hospital District and the Joint Ethical Committee of the Oulu University Hospital, respectively. The diagnosis of DD or HHD was based on clinical appearance and histological diagnosis. The patients gave their written consent. Four-millimeter skin biopsies were taken from apparently healthy skin and lesional areas in eight DD patients aged 35-69 years and nine HHD patients aged 45-80 years. In addition three patients with DD and three patients with HHD gave biopsies from lesional skin only. Paraffinembedded skin biopsies of four HHD patients and five DD patients were obtained from the Department of Pathology, Turku University Central Hospital. Three control samples were obtained from otherwise healthy patients undergoing plastic surgery. The fresh skin samples were frozen in liquid nitrogen or in isopentane cooled in liquid nitrogen and stored at $-70^{\circ} \mathrm{C}$ or in liquid nitrogen.

\section{Primary antibodies}

The following primary antibodies were used: affinity-purified rabbit polyclonal antibodies to human claudin-1 (51-9000), Z0-1 (61-7300), and occludin (71-1500); and mouse monoclonal antibodies to human claudin-4 (18-7341), Ecadherin (33-4000), and Z0-1 (33-9100), all from Zymed Laboratories Inc., South San Francisco, CA, USA. Mouse monoclonal antibody to $\beta$-catenin (M3539) was purchased from Dako (Glostrup, Denmark). Nonimmunized mouse (2025) and rabbit (2027) IgG were purchased from Santa Cruz Biotechnology Inc., (Santa Cruz, CA, USA). All antibodies against junctional proteins recognized intracellular epitopes.

\section{Indirect immunofluorescence labeling}

Punch biopsy samples were cut into $7 \mu \mathrm{m}$ cryosections, mounted on silanated glass slides, and fixed in $100 \%$ methyl alcohol at $-20^{\circ} \mathrm{C}$ for $10 \mathrm{~min}$. To prevent nonspecific binding, the samples were preincubated in $1 \%$ bovine serum albumin (BSA) in phosphate buffered saline (PBS) for $15 \mathrm{~min}$. Antibodies were diluted in $1 \%$ BSA-PBS, and incubated on the samples at $4^{\circ} \mathrm{C}$ for $20 \mathrm{~h}$. Either primary antibodies were used alone or antibodies raised in mouse and rabbit were mixed for double labeling. Following five 5-min washes in PBS, the samples were incubated with secondary antibodies and Hoechst nuclear stain (dilution 1:10,000) at $20^{\circ} \mathrm{C}$ for $1 \mathrm{~h}$. Secondary antibodies used were Alexa Fluor 568 conjugated goat anti-rabbit IgG (A11011) or Alexa Fluor 488 conjugated goat anti-mouse IgG (A11029) from Molecular Probes Inc. (Eugene, OR, USA). In double labeling the secondary antibodies for mouse and rabbit were mixed. The samples were washed in PBS, dipped in double-distilled water and mounted with Glycergel (Dako, Glostrup, Denmark). In control immunoreactions primary antibodies were replaced with $1 \%$ BSA-PBS or nonimmunized mouse or rabbit IgG.

\section{Avidin-biotin immunolabeling of paraffin-embedded tissues}

Formalin-fixed and paraffin-embedded skin specimens were immunolabeled with the avidin-biotin method. The sections were cut and mounted on SuperFrost Plus microscope slides (Menzel-Gläser; Braunschweig, Germany), deparaffinized, and hydrated in descending ethanol series. To retrieve TJ antigens Z0-1 and claudin-1, the samples were boiled for $10 \mathrm{~min}$ in a microwave oven in 10 $\mathrm{mM}$ Tris, $1 \mathrm{mM}$ ethylene diamine tetra-acetic acid (EDTA), pH 9, and subsequently cooled in the same solution at room temperature for 30 min. Endogenous peroxidase activity was quenched by treating the sections in $0.3 \% \mathrm{H}_{2} \mathrm{O}_{2}$ for $30 \mathrm{~min}$. To prevent nonspecific binding the sections were incubated in horse serum diluted in PBS. Antibodies to TJ were diluted in PBS supplemented with $1 \%$ BSA and incubated on the samples overnight at $4^{\circ} \mathrm{C}$. The bound antibodies were visualized using the appropriate avidin-biotin peroxidase kit (Vectastain; Vector Laboratories, Burlingame, CA, USA) with 3.3'-diaminobenzidine tetrahydrochloride (DAB) as a chromogen (DAB peroxidase substrate kit; Vector Laboratories). Sections were counterstained with Mayer's hematoxylin. In negative control reactions the primary antibody was replaced with 1\% BSA-PBS.

\section{Microscopy}

All examples of indirect immunofluorescence labeling were photographed using the confocal microscope. Confocal laser scanning microscopy was carried out using a Zeiss LSM 510 META confocal microscope equipped with argon-ion and helium-neon lasers (Zeiss; Jena, Germany) and LSM 3.0 software. The objectives were 40X (oil immersion, numeric aperture 1.3) and 63X (oil immersion, numeric aperture 1.4). For excitation, the 405-nm line was used for Hoechst, the 488-nm line for Alexa Fluor ${ }^{\mathrm{TM}} 488$, and the 543 -nm line for Alexa Fluor ${ }^{\mathrm{TM}} 568$ and Cy3. The images were saved in an LSM image browser program and exported to Adobe Photoshop in jpg format.

\section{TEWL measurements}

The VapoMeter with a closed cylindrical chamber (Delfin Technologies Ltd, Kuopio, Finland) was used for TEWL analyses. TEWL was measured from lesional skin of nine patients with HHD and eight patients with DD. Measurements were obtained from lesional and a corresponding healthy abdominal skin area of each patient. In HHD patients lesional areas included axillary (6), groin (1), leg (1), and chest (1) areas, while all lesional values from DD patients were measured from the chest (8) area. The average values were calculated for normal and lesional skin.

\section{Results}

To investigate the function of the epidermal barrier in HHD and DD, TEWL was measured in nine patients with HHD and eight patients with DD. The values of non-lesional and lesional skin were compared. The results showed that TEWL in lesional HHD and DD areas was increased four- and three-fold, respectively, compared to the normal skin. The average TEWL for non-lesional HHD skin was 12.4, while the average TEWL value for lesional skin was 44.6. In non-lesional DD skin the average TEWL value was 17.8 , while in the lesional skin the average value was 50.0. The $p$-values were calculated using the t-test for independent samples, and resulted in a $p$-value of $<0.01$ for both diseases (Figure 1).

The localization of TJ components claudins1 and $-4, \mathrm{ZO}-1$, and occludin in frozen and paraffin-embedded skin of 14 cases of HHD and 14 cases of DD was demonstrated using indirect immunofluorescence and avidinbiotin immunolabeling. The results showed that the localization of $\mathrm{TJ}$ proteins in the healthy looking epidermis in both diseases was similar to that of the control skin. The expression of $\mathrm{TJ}$ proteins remained normal in the vicinity of the lesions.

In HHD lesions typical suprabasal blistering was observed. Groups of acantholytic cells were noted in the blister fluid (Figure 2a-e). In DD lesions characteristic acanthosis and acan- 
tholysis were detected (Figure 2f-j). In HHD and DD samples Z0-1 was located in the blister roof (Figure $2 \mathrm{~b}, \mathrm{~g}$ ). In addition, in HHD ZO1 was seen in the remaining cell-cell contacts of acantholytic spinous cells (Figure 2b). Occludin was present in the intercellular junctions of the granular cell layer located in the blister roof (Figure 2c, h). Claudin-1 was detected in all living cell layers (Figure 2d, i), the basal cell layer being only faintly labeled. Claudin-4 was localized mainly in the upper epidermis (Figure 2e, j). To conclude, the general distribution of TJ proteins occludin, and claudin-1 and -4 corresponded to that described earlier for normal skin. Thus the acanthosis of DD could not be shown to induce spreading of TJ to the spinous cell layers.

To correlate the dynamics of $\mathrm{TJ}$ proteins with that of desmosomal and adherens junction proteins, we double-labeled the sections with antibodies to TJ proteins and desmoplakin, $\beta$-catenin, or E-cadherin. The antibody recognizing the intracellular part of E-cadherin showed labeling of the intercellular contacts in the acantholytic cells (Figure 2c, h). This finding was in accordance with a previous study. ${ }^{16}$ Desmoplakin was co-localized with claudin-1 in the granular cell layer (Figure 2d, i). In acantholytic cells desmoplakin showed a diffuse cytoplasmic pattern while claudin-1 stayed in the remaining intercellular contacts (Figure 2d). The co-localization of TJ proteins with $\beta$-catenin, desmoplakin, and E-cadherin in acantholytic cells was studied in more detail in the HHD lesions (Figure 3). Double labeling for $\mathrm{ZO}-1$ and $\beta$-catenin revealed the presence of both proteins in the cell periphery, although some cytoplasmic Z0-1 labeling was noted as well (Figure 3a). Double-labeling for claudin-1 with $\beta$-catenin revealed localization of both proteins at the plasma membrane (Figure $3 \mathrm{~b}$ ). Plenty of cytoplasmic $\beta$-catenin was seen in acantholytic cells (Figure $3 \mathrm{a}, \mathrm{b}$ ), thus suggesting that claudin- 1 and $\beta$-catenin follow different patterns in acantholysis. In addition to being present in intercellular contacts, claudin-1 was seen in the periphery of some cells without an apparent contacting cell (Figure 3c). Double labeling of claudin-1 and desmoplakin also demonstrated different dynamics of these proteins: claudin-1 remained at the plasma membrane of the intercellular contacts while desmoplakin was diffusely distributed in the cytoplasm. In acantholysis the cells gradually lost contacts with almost all the neighboring cells. During this process claudin-1 finally disappeared from the plasma membrane. However no clear cytoplasmic redistribution of claudin-1 could be seen. Claudin-1 co-localized with E-cadherin in the granular cell layer as well as in some intercellular contacts of the acantholytic cells (Figure $3 d)$.

\section{Barrier function in resting state}
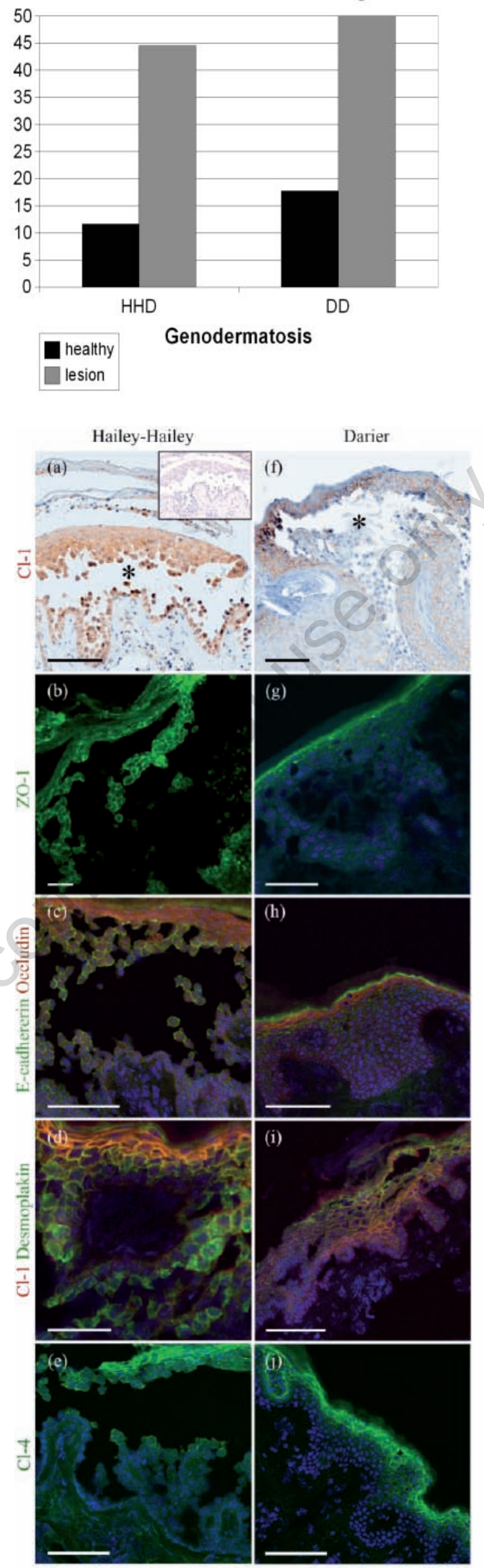

Figure 1. Average TEWL values of nine patients with HaileyHailey disease (HHD) and eight patients with Darier's disease (DD). Lesional skin shows three- to four-fold higher TEWL values compared to the non-lesional skin. HHD nonlesional (12.4) compared to lesional (44.6): $p<0.01 ; \mathrm{DD}$ non-lesional $(17.8)$ compared to lesional (50.0): $p<0.01$.
Figure 2. Hailey-Hailey disease (HHD) (a-e) and Darier's disease (DD) (f-j) lesions immunolabeled for tight junction proteins claudin-1 (a, f), ZO-1 (b, g), and claudin-4 (e, j); double labeling for E-cadherin and occludin (c, h), and desmoplakin and claudin-1 $(d, i)$. In (a) the inset is a control without any primary antibody. Avidin-biotin immunolabeling visualizes the suprabasal blisters (asterisks) in lesions of both diseases ( $a$, f). Claudin-1 is expressed in all epidermal cell layers (a, f). ZO-1 localizes to the upper epidermis and acantholytic cells in the HHD blister (b) while in DD, ZO-1 is expressed only in the granular cell layer (g). Occludin (red) is restricted to the granular cell layer in both diseases, while Ecadherin (green) is seen in intercellular junctions in all epidermal cell layers (c, h). Claudin-1 and desmoplakin are visible in all epidermal cell layers (d, i). Claudin-4 localizes to the upper epidermis and acantholytic cells in the Hailey-Hailey blister and in DD (e, j). Scale bar: $100 \mu \mathrm{m}$ in (a), (c), (e), (f), (h), (i) and (j); $50 \mu \mathrm{m}$ in (b), (d) and (g). 


\section{Discussion}

Based on genetically engineered mouse models, TJs are known to contribute to the epidermal barrier by regulating the TEWL. ${ }^{9}$ In the present study, TEWL was measured in normal and lesional HHD and DD skin. The results showed that TEWL in lesional areas was increased four- and three-fold, respectively, compared to the non-lesional skin, although the granular cell layer in the blistering area had all the elements needed for complete TJs; namely Z0-1, occludin, and two claudins. Excess evaporation of water apparently takes place through the broken blisters.

The formation of $\mathrm{TJ}$ in vitro is dependent on external calcium concentration. ${ }^{7,8,15}$ Here we investigated $\mathrm{TJ}$ in diseases in which the epidermal calcium gradient is aberrant, which might have an impact on the expression of TJ proteins. The results showed that $\mathrm{ZO}-1$ was present in acantholytic suprabasal cells unlike in non-lesional epidermis, where Z0-1 was detected only in the granular cell layer. The expression of all the other TJ proteins studied remained essentially the same in the tissue level compared to the non-lesional skin. Thus the expression of Z0-1 can be speculated to show different regulation compared to the other TJ components studied. Aberrant epidermal differentiation previously has been shown to change the expression pattern of TJ proteins, Z0-1 in particular. Specifically healing of an experimental wound leads to epidermal hypertrophy, which displays an intense expression of Z0-1. ${ }^{14}$ In psoriasis acanthosis is associated also with expression of ZO-1 in the spinous cell layers. ${ }^{13}$ In contrast acanthosis of DD epidermis was characterized with the lack of ZO-1 in the spinous cells. This suggests that the epidermal keratinocytes of DD follow different regulation of $\mathrm{TJ}$ components compared to psoriasis. In addition the expression of $\mathrm{ZO}$ 1 can be seen as a sensitive indicator of abnormal differentiation.

This study compared the dynamics of TJ proteins with desmosomal and adherens junction proteins at a single cell level. Previously it has been shown that intra- and extracellular domains of desmosomal cadherins and E-cadherin dissociate, and desmoplakin is internalized in acantholytic cells in HHD and DD., ${ }^{46}$ The present evidence suggests that TJ components claudin-1, Z0-1, and occludin have somewhat different dynamics in acantholysis compared to desmosomal and adherens junction proteins. For instance, desmoplakin exhibited a diffuse cytoplasmic distribution in the acantholytic cells while TJ proteins claudin-1 and occludin remained in the cell-cell contact sites. Claudin1 was seen even in the free cell border of some cells that were not in contact with neighboring cells. The absence of cytoplasmic claudin-1 in
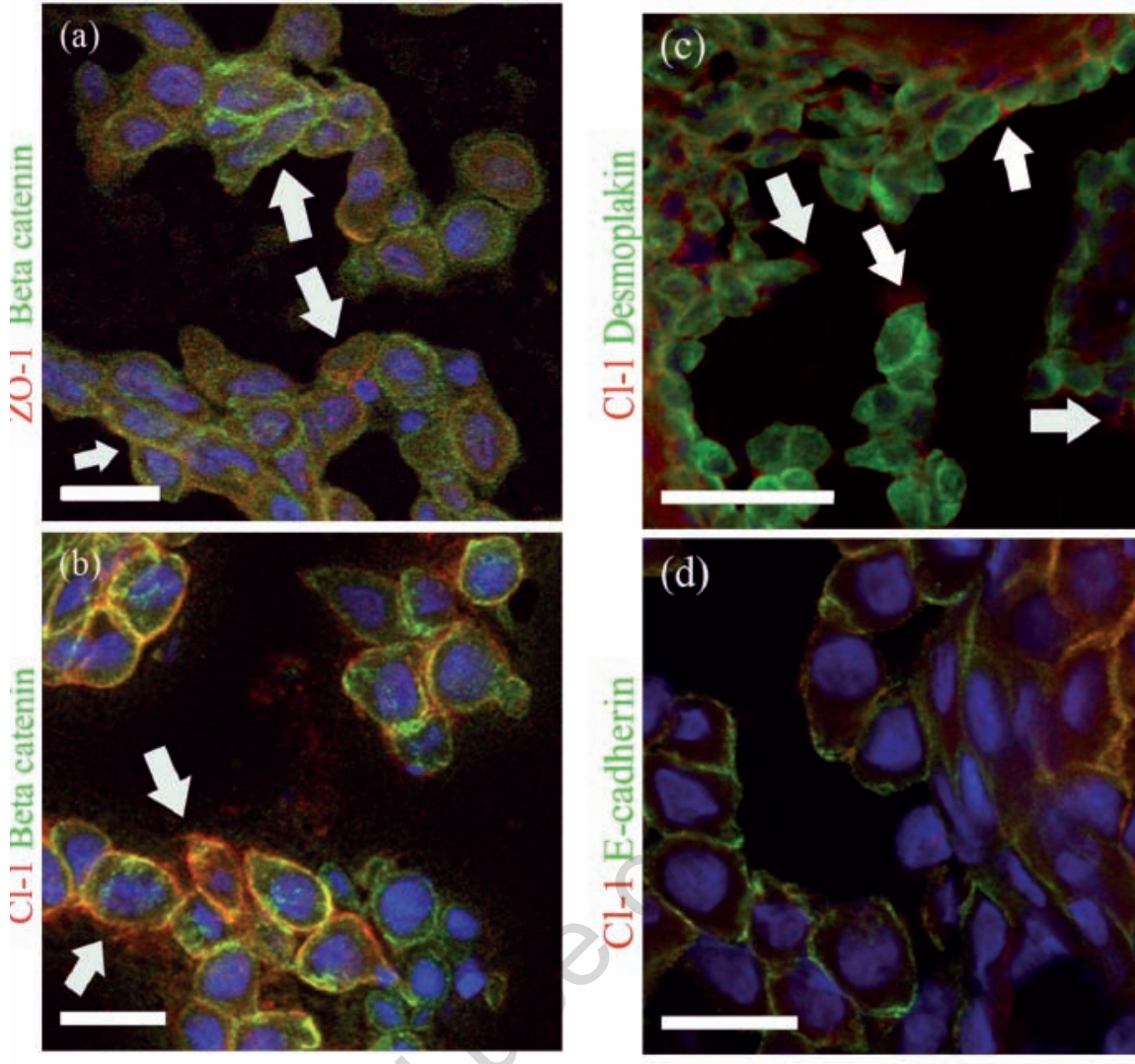

Figure 3. Acantholytic cells in Hailey-Hailey disease (HHD) lesions, double immunolabeled for TJ proteins and adherens junction or desmosomal components. ZO-1 (red) and $\beta$-catenin (green) are present in some of the remaining intercellular junctions, while both proteins can be detected intracellularly and in plasma membranes not in contact with neighboring cells (arrows) (a). Claudin-1 and $\beta$-catenin in the cell-cell contacts (b). Claudin-1 is seen in the plasma membranes of cells not in contact with the neighboring cells (arrows) (b,c). Double labeling for desmoplakin (green) and claudin -1 (red) shows internalization of desmoplakin while claudin-1 stays in the plasma membrane (c). E-cadherin (green) and claudin-1 (red) are present at the plasma membrane (d). Scale-bar: 20 $\mu \mathrm{m}$ in (a), (b), (d); $50 \mu \mathrm{m}$ in (c).

the acantholytic cells may be because of the fact that it remains in the cell border or it is rapidly degraded if internalized. The intracellular plaque protein Z0-1 was present in the remaining intercellular contacts of the acantholytic cells, but was not detectable in the free cell borders. In contrast to claudin-1, some cytoplasmic Z0-1 could be seen.

To conclude, in HHD and DD lesions the abnormalities in the calcium gradient and epidermal differentiation have little effect on the expression of TJ proteins at the tissue level, while the acantholytic process merely has an impact on the dynamics of existing proteins at the single cell level.

\section{References}

1. Sudbrak R, Brown J, Dobson-Stone C, et al. Hailey-Hailey disease is caused by muta- tions in ATP2C1 encoding a novel $\mathrm{Ca}(2+)$ pump. Hum Mol Genet 2000;9:1131-40.

2. Hu Z, Bonifas JM, Beech J, et al. Mutations in ATP2C1, encoding a calcium pump, cause Hailey-Hailey disease. Nat Genet 2000;24:61-5.

3. Sakuntabhai A, Ruiz-Perez V, Carter S, et al. Mutations in ATP2A2, encoding a $\mathrm{Ca} 2+$ pump, cause Daries disease. Nat Genet 1999;21:271-7.

4. Burge SM, Garrod DR. An immunohistological study of desmosomes in Darier's disease and Hailey-Hailey disease. $\mathrm{Br} \mathrm{J}$ Dermatol 1991;124:242-51.

5. Foggia L, Hovnanian A. Calcium pump disorders of the skin. Am J Med Genet C Semin Med Genet 2004;131C:20-31.

6. Leinonen PT, Hägg PM, Peltonen S, et al. Re-evaluation of the normal epidermal calcium gradient, and analysis of calcium levels and ATP receptors in Hailey-Hailey and Darier epidermis. J Invest Dermatol 2009; 129:1379-87. 
7. Pummi K, Malminen M, Aho $\mathrm{H}$, et al. Epidermal tight junctions: $\mathrm{ZO}-1$ and occludin are expressed in mature, developing, and affected skin, and in vitro differentiating keratinocytes. J Invest Dermatol 2001;117:1050-8.

8. Brandner JM, Kief S, Grund C, et al. Organization and formation of the tight junction system in human epidermis and cultured keratinocytes. Eur J Cell Biol 2002;81:253-63.

9. Furuse M, Hata M, Furuse $\mathrm{K}$, et al. Claudin-based tight junctions are crucial for the mammalian epidermal barrier: a lesson from claudin-1-deficient mice. J Cell Biol 2002;156:1099-111.

10. Turksen K, Troy TC. Permeability barrier dysfunction in transgenic mice overexpressing claudin 6. Development 2002;129:
1775-84.

11. Troy TC, Rahbar R, Arabzadeh A, et al. Delayed epidermal permeability barrier formation and hair follicle aberrations in Inv-Cldn6 mice. Mech Dev 2005;122:80519.

12. Morita K, Miyachi Y. Tight junctions in the skin. J Dermatol Sci 2003;31:81-9.

13. Peltonen S, Riehokainen J, Pummi K, et al. Tight junction components occludin, Z0-1, and claudin-1, -4 and -5 in active and healing psoriasis. Br J Dermatol 2007;156:46672.

14. Malminen M, Koivukangas V, Peltonen J, et al. Immunohistochemical distribution of the tight junction components Z0-1 and occludin in regenerating human epidermis. Br J Dermatol 2003;149:255-60.

15. Yuki T, Haratake A, Koishikawa H, et al.
Tight junction proteins in keratinocytes: localization and contribution to barrier function. Exp Dermatol 2007;16:324-30.

16. Hakuno M, Shimizu H, Akiyama M, et al. Dissociation of intra- and extracellular domains of desmosomal cadherins and Ecadherin in Hailey-Hailey disease and Darier's disease. Br J Dermatol 2000;142: 702-11.

17. Muller SL, Portwich M, Schmidt A, et al. The tight junction protein occludin and the adherens junction protein alpha-catenin share a common interaction mechanism with Z0-1. J Biol Chem 2005;280:3747-56.

18. Tinkle CL, Pasolli HA, Stokes N, et al. New insights into cadherin function in epidermal sheet formation and maintenance of tissue integrity. Proc Natl Acad Sci USA 2008;105:15405-10. 